\title{
False memories for compound words: Role of working memory
}

\author{
MARK TIPPENS REINITZ \\ University of Puget Sound, Tacoma, Washington \\ and \\ SHARON L. HANNIGAN \\ St. Lawrence University, Canton, New York
}

\begin{abstract}
Subjects studied pairs of compound words; pair members were presented simultaneously (one above the other) for 2 sec or sequentially (one immediately following the other) for $1 \mathrm{sec}$ each, and 6-sec interstimulus intervals separated the end of presentation of one pair and the start of that of another. A subsequent recognition test included within-pair and between-pair conjunction foils (recombinations of stimulus parts from the same study pair and from separate pairs, respectively). Previous experiments using faces as stimuli have demonstrated that when faces are presented simultaneously there are many more false alarms to within-pair than to between-pair conjunction items, and when faces are presented sequentially there is an equal number of false alarms in those two conditions. However, Experiment 1 showed that for compound word stimuli there were equally high false alarm rates to both types of foils in both study conditions relative to completely new test items. Experiment 2 showed that when rehearsal of compound words was prevented, the pattern of conjunction errors was very similar to the one typically obtained for faces. In Experiment 3, subjects falsely recalled conjunctions of withinpair compound words but not conjunctions of between-pair words in the simultaneous-study condition; no conjunctions were recalled in the sequential-study condition. The results support the idea that working memory processing is necessary for binding stimulus parts together in episodic memory.
\end{abstract}

A long line of research indicates that stimuli and events are encoded into memory as sets of parts. For instance, subjects unknowingly integrate various units of information into their recollections of events, including their own world knowledge (see, e.g., Bower, Black, \& Turner, 1979; Hannigan \& Reinitz, 2001) and information experienced in other episodes (see, e.g., Albert, Reinitz, Beusmans, \& Gopal, 1999; Hannigan \& Reinitz, 2003; E. F. Loftus, Miller, \& Burns, 1978). Thus, memories for events are constructed from various units of information residing in memory. There is evidence that individual objects are also encoded as sets of parts; for instance, Brown and McNeill (1966) showed that subjects may recall some aspects of a word (e.g., its first letter or number of syllables) without being able to recall the entire word. Zechmeister and McKillip (1972) showed that subjects may recall the location on a page where specific information had occurred without being able to remember the information itself, again indicating the multidimensional nature of representations for simple stimuli. Furthermore, parts of separate stimuli may sometimes be remembered

We thank Danny Concha for testing subjects in the pilot experiment that led to this study. We also thank Yonatan Goshen-Gottstein, Todd Jones, Kathleen McDermott, and an anonymous reviewer for helpful comments on a draft of this paper. Address correspondence to M. T. Reinitz, Department of Psychology, University of Puget Sound, 1500 North Warner, Tacoma, WA 98416 (e-mail: mreinitz@ups.edu). as having occurred in a single stimulus (see, e.g., Reinitz, Lammers, \& Cochran, 1992; Underwood, Kapelak, \& Malmi, 1976). Finally, most formal models of memory represent stimuli as sets of features (see, e.g., Hintzman, 1988; McClelland \& Rumelhart, 1985; Metcalfe, 1990; Murdock, 1982), demonstrating the utility of parts-based representations for items and events.

If events and objects are encoded into memory as sets of parts, then it is necessary for those parts to be bound together in memory so that parts that had occurred together are subsequently remembered together. This binding process has been the topic of much recent research and has been proposed to be a central function of the hippocampus (see, e.g., Chalfonte \& Johnson, 1996; Cohen \& Eichenbaum, 1993; Kroll, Knight, Metcalfe, Wolf, \& Tulving, 1996; Reinitz, Verfaellie, \& Milberg, 1996). More specifically, these authors have proposed that the function of the hippocampus is to encode episodic information by creating new associations between preexisting units stored in memory, rather than to encode novel units into memory. Evidence to support this comes from studies showing that patients with hippocampal damage can sometimes recognize previously presented stimulus components (e.g., they may recognize STAR as old if they recently studied the word STARGAZE) while being unable to discriminate old stimuli from new ones constructed from previously presented parts (e.g., being unable to discriminate whether STARGAZE or STARFISH is old after hav- 
ing recently studied STARGAZE and CATFISH; Kroll et al., 1996, Reinitz et al., 1996).

In addition, there is substantial evidence that working memory processing mediated by prefrontal cortex plays a central role in binding together parts of stimuli so that they are remembered together later (for discussions, see Moscovitch, 2000; Reinitz, 2002). For instance, dividing conscious attention in normal subjects (by introducing a secondary task) produces the pattern just described as occurring following hippocampal damage: Subjects can reject partly or completely new stimuli (presumably because these contain new features, and dividing attention does not eliminate feature encoding), but they are unable to discriminate old stimuli from new stimuli constructed entirely of old parts (see, e.g., Reinitz, Morrissey, \& Demb, 1994). Various forms of evidence indicate that division of attention interferes specifically with prefrontal executive functions associated with working memory (see, e.g., Iidaka, Anderson, Kapur, Cabeza, \& Craik, 2000). In addition, recent studies of aging have demonstrated increasing binding deficits with decreasing availability of dopamine, which mediates prefrontal executive processing (e.g., Braver et al., 2001; Schacter, Kaszniak, Kihlstrom, \& Valdiserri, 1991). These findings imply that prefrontal executive (working memory) functions create associations between representations of stimulus parts in memory, so that those parts are subsequently remembered together.

Reinitz and Hannigan (2001) recently reported results consistent with the proposal that working memory creates associations between stimulus components that are then encoded into episodic memory. Those authors used a memory conjunction error paradigm (see, e.g., Reinitz et al., 1992) to investigate binding. Eight pairs of different faces were presented during the study phase of a recognition experiment. For some subjects, the two faces within a study pair were presented simultaneously, one above the other, for $8 \mathrm{sec}$. For other subjects, the members of each study pair were presented sequentially for $4 \mathrm{sec}$ each, with the second pair member appearing immediately following the offset of the first pair member. A long (10-sec) interstimulus interval (ISI) separated the end of one pair and the start of the next, so that both simultaneously presented and sequentially presented items were subjectively presented in pairs. This simultaneous/ sequential-study manipulation had a profound effect on the pattern of errors in the subsequent "old"/"new" recognition test. The test included two types of conjunction foils, which are new items constructed by recombining parts of previously studied items: A conjunction test face contained the hair and mouth of one previously studied face and the eyes and nose of another. Within-pair conjunction faces were constructed from parts of parent faces that had occurred in the same study pair, and betweenpair conjunction faces were constructed from parent faces that had occurred in separate study pairs. Subjects in the simultaneous-study condition had substantial difficulty discriminating old faces from within-pair con- junction faces, as indicated by the very high false alarm rate to within-pair conjunction faces in that condition. Subjects in this condition made few false alarms to between-pair conjunction items. That is, when faces were studied simultaneously, subjects were subsequently quite good at remembering which study pair contained specific facial features (as indicated by the low betweenpair conjunction error rate) but had difficulty remembering which face within the pair contained specific features (as indicated by the high within-pair conjunction error rate). Subjects who studied the faces sequentially made fewer false alarms to within-pair conjunction faces and more false alarms to between-pair conjunction items than did subjects who studied the faces simultaneously. Moreover, subjects in the sequential-study condition had approximately equal false alarm rates for between-pair and within-pair conjunction faces.

One possible explanation for the effects of simultaneousversus sequential-study presentations is that when items are presented simultaneously, all of their features are simultaneously present in working memory. When items are presented sequentially, only one item at a time is present in working memory. Items that are simultaneously present in working memory may be indiscriminately interassociated with one another, so that strong association strengths between parts of simultaneously presented items are formed. Such a binding mechanism would have the benefit of integrating the various aspects of a momentary event so that they are remembered together, but might have the cost of making it difficult to segregate the parts of simultaneously studied stimuli into separate memories of the individual items. By this explanation, parts of sequentially studied faces were interassociated with other within-face parts, but not with parts of the other face in the study pair, because subjects were simultaneously aware only of the parts of individual faces. However, parts of faces in the simultaneous-study condition were interassociated both with other parts of the same face and with parts of the other face in the pair, because the two faces occupied working memory simultaneously. To put it differently, sequential-study subjects may have encoded each face as a separate list item, and simultaneous-study subjects may have encoded each face pair as an individual list item. In consistency with this explanation, simultaneous-study subjects made fewer between-pair conjunction errors. This is expected because recognition errors increase rapidly with number of list items (Strong, 1912).

The purpose of the present research was to test the adequacy and generality of the proposal that stimulus features that are concurrently within working memory may become highly interassociated, making it difficult subsequently to assign those parts to the specific stimuli that had originally contained them. When faces are simultaneously presented, there are many more within-pair than between-pair conjunction errors, and when they are presented sequentially there are approximately equal numbers of within-pair and between-pair conjunction errors. 
In Experiment 1, we tested whether the same pattern of errors is obtained for simultaneously presented words and not for sequentially presented words. There are many differences between our face stimuli and words; for instance, the faces are pictorial, unfamiliar, lack semantic content, and do not have preexisting memory representations. Words are nonpictorial, familiar, have meanings, and have preexisting memory representations. Given these differences, the finding of identical study condition effects for the two stimulus types would imply a very general role of working memory in binding. In Experiment 2, we introduced a secondary task to test the effects of disrupting working memory involvement during the study phase for compound word stimuli. Finally, in Experiment 3, we examined recall rather than recognition, to test the generality of our results across memory tests.

\section{EXPERIMENT 1}

In Experiment 1, we tested whether there are more within-pair than between-pair conjunction errors when compound words are studied simultaneously, and not when they are studied sequentially. Subjects studied a series of compound-word pairs. Words within each pair were presented either simultaneously (one above the other) or sequentially. The subjects then received a recognition test containing old words, within-pair conjunction words, between-pair conjunction words, and completely new words.

There is empirical evidence that suggests that different processes may give rise to false alarms to conjunction stimuli for pictures and words. For instance, Chandler and Gargano (1998) showed that there is more interference between pictures when both pictures are presented during the study phase of a recognition experiment than when one picture is presented during the study phase and the interfering picture is presented during the test phase. For words, the result was quite different: There was more interference when one word pair was presented during the study phase and the interfering pair was presented during the test than when both were presented during the study phase. This implies that different underlying mechanisms may produce interference for words and for pictures. False alarms to conjunction items constitute an interference effect, in which the presence in memory of multiple, similar stimulus items gives rise to incorrect responses on a memory test. Given these differences, it is important to test whether the patterns of results obtained for faces also occur for words.

\section{Method}

Subjects. Forty-eight Boston University undergraduates participated for credit in their introductory psychology classes. They were tested in 16 groups of 3 subjects each.

Stimuli and Apparatus. The stimuli were 24 compound words that constituted eight triads from the stimulus set devised by Reinitz and Demb (1994). Each triad contained three compound words in such a way that the first two members of the triad could be recom- bined to create the third member (e.g., BLACKTOP, HEADBOARD; BLACKBOARD). Four additional, unrelated compound words served as primacy and recency study items. The stimuli were projected onto a screen by Kodak slide projectors equipped with tachistoscopic shutters.

Design and Procedure. During the study phase, the subjects viewed a series of 14 compound words presented as seven word pairs. For half of the subjects, the two words appeared simultaneously, one just above and the other just below a central fixation dot (simultaneous-study condition). Each simultaneous-study trial was $2 \mathrm{sec}$ long. The remaining subjects were in the sequential-study condition. Here, words were presented centrally for $1 \mathrm{sec}$ each; the second member of the pair was presented $500 \mathrm{msec}$ after the offset of the first member. In both study conditions, there was a 6-sec ISI between the end of presentation of one study pair and the start of that of the next. This relatively long interval was chosen to ensure that the subjects perceived the two stimuli as occurring within a distinct pair. Only word pairs in the second, third, fourth, fifth, and sixth study positions were used to create test stimuli; the first and seventh study pairs served as primacy and recency control items and were not tested. We chose to use a relatively short (14-item) study list to maximize explicit recollection of the study items, as we have proposed that effects of simultaneous study reflect binding errors in episodic memory. In this and both subsequent experiments, the subjects were told prior to the study session that they would later receive a memory test for the words they were about to see.

Following the study session, the subjects performed a $10-\mathrm{min}$ mental rotations test as a filler task. They then received an eightitem recognition test containing four types of test items: old words, which had been presented in the study session; within-pair conjunction words, which were recombinations of parts of words that had been studied in a single study pair; between-pair conjunction words, which were recombinations of words from study pairs that were separated by an intervening study pair; and new words, which did not contain any previously presented word segments. Two test items were presented in each of the four test conditions. The test words were presented one at a time for $4 \mathrm{sec}$ each. The subjects were told to circle the word old on a response sheet if the test word was identical to a single previously studied word, and to circle the word new otherwise.

Two separate sets of stimuli were used to reduce the likelihood of any stimulus-specific effects. Twenty-four subjects received one stimulus set, and the remaining subjects received the other stimulus set. All of the subjects who received a given stimulus set received the same eight test items. The test condition order was random. The goal of this and the following experiments was to compare false alarm rates for within- and between-pair conjunction words across simultaneous- and sequential-study conditions. Therefore, the stimuli were presented equally often in the two conjunction conditions, and the same set of study and test orders was used across the study conditions. To counterbalance the test stimuli across the conjunction conditions, two different study orders were used for each stimulus set. A test word that appeared in the within-conjunction condition for the first study order appeared in the between-conjunction condition for the second study order, and vice versa. It was not possible to sample all of the study positions equally during construction of the test items. Instead, the two between-pair conjunction items were always constructed from parent items that appeared in Study Positions 2 and 4 and Study Positions 4 and 6. Within-pair conjunction items were always drawn from Study Positions 3 and 5, and old items were always drawn from Study Positions 2 and 6. For every simultaneous-study group, there was a sequential-study group that received the same study and test words in the same order. Conjunction test items were always constructed from one word that had appeared in the top position of a study pair and from another word that had appeared in the bottom position. The complete set of experimental stimuli is shown in Table 1, which also shows how items 
Table 1

List of Experimental (Nonfiller) Words Used in Experiments 1-3

\begin{tabular}{|c|c|c|}
\hline Test Condition & Test Word & Studied Words \\
\hline \multicolumn{3}{|c|}{ Stimulus Set 1 (Groups 1 and 2) } \\
\hline Old & $\begin{array}{l}\text { eyelid } \\
\text { wineglass }\end{array}$ & $\begin{array}{l}\text { eyelid } \\
\text { wineglass }\end{array}$ \\
\hline W-Con & $\begin{array}{l}\text { starfish } \\
\text { handgun }\end{array}$ & $\begin{array}{l}\text { stargaze, catfish } \\
\text { handstand, shotgun }\end{array}$ \\
\hline B-Con & $\begin{array}{l}\text { lighthouse } \\
\text { classmate }\end{array}$ & $\begin{array}{l}\text { lightweight, clubhouse } \\
\text { classwork, shipmate }\end{array}$ \\
\hline New & $\begin{array}{l}\text { cupcake } \\
\text { dumbbell }\end{array}$ & $\begin{array}{l}- \\
-\end{array}$ \\
\hline \multicolumn{3}{|c|}{ Stimulus Set 2 (Groups 3 and 4 ) } \\
\hline Old & $\begin{array}{l}\text { doorknob } \\
\text { coffeepot }\end{array}$ & $\begin{array}{l}\text { doorknob } \\
\text { coffeepot }\end{array}$ \\
\hline W-Con & $\begin{array}{l}\text { toothache } \\
\text { newspaper }\end{array}$ & $\begin{array}{l}\text { toothpaste, heartache } \\
\text { newsworthy, wastepaper }\end{array}$ \\
\hline B-Con & $\begin{array}{l}\text { blackboard } \\
\text { seashore }\end{array}$ & $\begin{array}{l}\text { blacktop, headboard } \\
\text { seafood, offshore }\end{array}$ \\
\hline New & $\begin{array}{l}\text { raincoat } \\
\text { tablespoon }\end{array}$ & $\begin{array}{l}- \\
-\end{array}$ \\
\hline
\end{tabular}

Note-W-Con, within-pair conjunction condition; B-Con, betweenpair conjunction condition. W-Con items for Group 1 served as B-Con items for Group 2, and vice versa. W-Con items for Group 3 served as B-Con items for Group 4, and vice versa. Test words were presented one at a time. Although studied words are presented side by side in this table, they were not presented side by side during the study phase.

were assigned to the various conditions. Eight groups of subjects were tested in the simultaneous condition, and eight groups were tested in the sequential condition. Four groups in each condition received Stimulus Set 1 and four groups received Stimulus Set 2.

\section{Results and Discussion}

The proportions of "old" responses for each of the four test conditions in each of the study conditions are shown in Figure 1. An analysis of variance (ANOVA) showed that there was a significant main effect of test condition $\left[F(3,138)=40.05, M S_{\mathrm{e}}=0.18, p<.01\right]$ but not of study condition $\left[F(1,46)=0.06, M S_{\mathrm{e}}=0.19\right]$. There was no evidence of a test condition $\times$ study condition interaction $\left[F(3,138)=0.78, M S_{\mathrm{e}}=0.18\right]$, even when the interaction was tested with a $2 \times 2$ ANOVA in which the old and new test conditions were removed from the analysis $\left[F(1,46)=.11, M S_{\mathrm{e}}=0.10\right]$.

There are two notable aspects of the results. First, essentially identical patterns of means were obtained in the two study conditions. In both cases, the subjects made many more false alarms to within-conjunction words than to between-conjunction words. This is in contrast with results of experiments that have employed faces as stimuli; in those cases, higher within-pair than betweenpair conjunction false alarm rates have been found only when stimuli are studied simultaneously. This finding may therefore indicate that different underlying mechanisms give rise to patterns of conjunction errors for faces and for words.

The second notable finding is the very high false alarm rate to within-pair conjunction words. In both study conditions, there is a high hit rate, and the false alarm rate for completely new words is at or near 0 . Nonetheless, the subjects made false alarms to within-pair conjunction words more than $40 \%$ of the time. This is a higher conjunction-error rate than has been observed in other

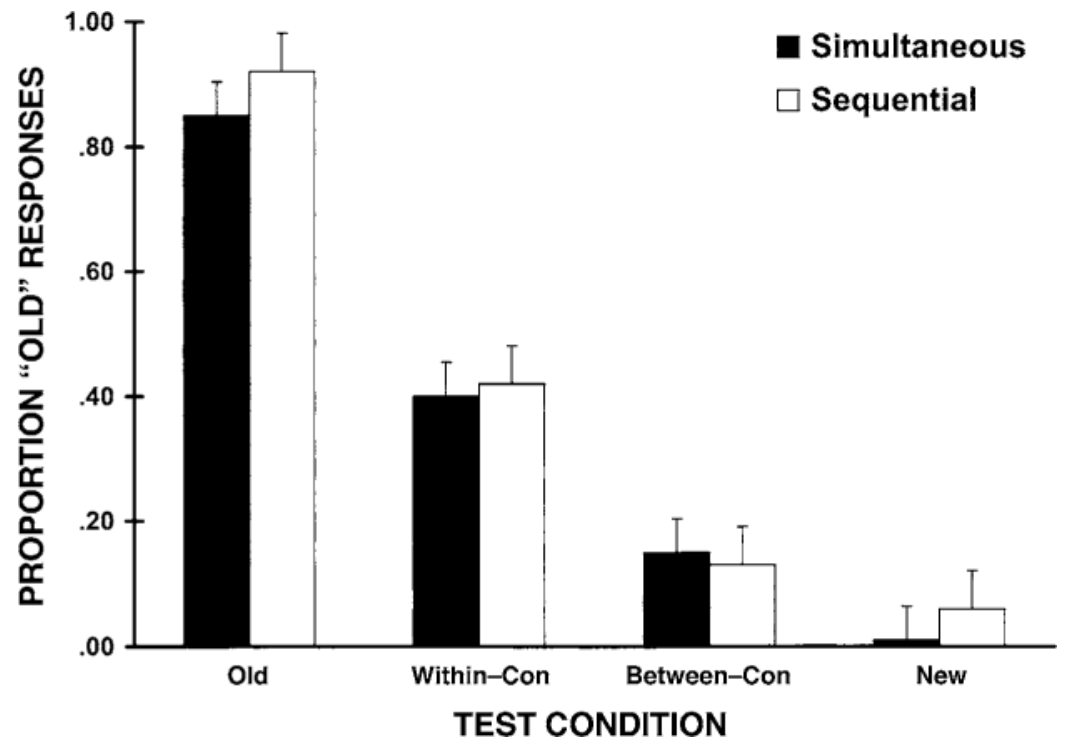

Figure 1. Results of Experiment 1: Proportion of "old" responses in each of the four test conditions for the two study conditions. The error bars show the $95 \%$ withinsubjects confidence intervals (G. R. Loftus \& Masson, 1994). Within-Con and Between-Con refer to the within-pair and between-pair conjunction conditions, respectively. 
experiments in which compound words have been employed as stimuli (e.g., Jones \& Jacoby, 2001), and is particularly surprising given the relatively small number of studied words and the relatively short retention interval. No previous experiments have presented compound words in pairs; the results indicate that pairing study words gives rise to substantial confusion regarding the way in which within-pair word components had been interrelated during the study phase.

Stimuli were counterbalanced across the conjunction conditions, but those items were not presented in the old or the new condition. Although our conclusions concern primarily differences between the two conjunction conditions, we wondered whether the results in the old and new conditions might be due to the specific words that were used in those conditions. To test for these stimulusspecific effects, we performed an ANOVA in which we treated stimulus set as a between-subjects factor. There was no statistical evidence of a main effect or any interaction effects involving stimulus set (all $F \mathrm{~s}<1$, all $p \mathrm{~s}>$ $.5)$. Thus, the identical pattern of results occurred when two completely different sets of stimuli were used, making it less likely that the results in the old and new conditions were due to the specific stimuli used.

Our failure to obtain differential patterns of responding in the simultaneous- and sequential-study conditions differs from results that we have repeatedly obtained for faces. In postexperiment interviews, many of the subjects reported intentionally using mnemonic strategies to interrelate words within each study pair. Words can remain in working memory for an extended period following their offsets by virtue of being simple, well learned, and meaningful. As a result, sequentially presented words can coexist simultaneously in working memory, and subjects can intentionally interrelate them. In contrast, it would be very hard for subjects to retain a conscious representation of an unfamiliar face during the presentation of the next study face, because faces consist of visual information that is perceptually complex and devoid of meaning. Given our proposals regarding the role of working memory in binding, we would expect more withinpair than between-pair conjunction errors whenever subjects were simultaneously aware of within-pair items. It is therefore possible that the higher within-pair than betweenpair conjunction false alarm rate that we have obtained for sequentially presented compound words occurred because representations of the first word within each pair were active in working memory during the presentation of the second word in each pair. In Experiment 2, we tested this possibility.

\section{EXPERIMENT 2}

In Experiment 1, the subjects made more false alarms to within-pair than to between-pair conjunction words in the sequential-study condition. In Experiment 2, we tested the possibility that this was due to the prolonged activation of the first word's representation in working mem- ory during the presentation of the second word in the pair. The experiment was identical to Experiment 1 except for the addition of a digit-monitoring task during the study phase. Digits were presented at a rate of $1 / \mathrm{sec}$ over a loudspeaker, whereas study words were presented visually; the subjects had to count the even numbers presented over the loudspeaker and report the total at the end of the study phase. This task was assumed to engage working memory sufficiently to prevent the subjects from maintaining a conscious representation of the first word in a sequentially presented pair during the presentation of the second pair member. If the false-alarm rate difference observed in the sequential-study condition of Experiment 1 was due to controlled processing mediated by prefrontal cortex, then there should not be a higher within-pair than between-pair conjunction false alarm rate in Experiment 2. The subjects would be simultaneously aware of both words in the simultaneous-study condition regardless of the digit-monitoring task. We therefore expected more within-pair than between-pair conjunction errors in the simultaneous-study condition.

\section{Method}

Subjects. Forty-eight Boston University undergraduates participated for credit in their introductory psychology classes. They were again run in groups of 3 . None of them had participated in the previous experiment.

Stimuli and Apparatus. The same set of word stimuli and apparatus used in Experiment 1 were used here. In addition, a series of random spoken digits was recorded onto a cassette tape. Digits were spoken at a rate of $1 / \mathrm{sec}$ in a female voice. The recording was slightly less than $1 \mathrm{~min}$ long. The tape was played using a portable cassette player at a volume loud enough to be easily heard by the subjects.

Design and Procedure. The same design and procedure (including the same set of study and test orders) that were used in Experiment 1 were used here, except for the inclusion of the digitmonitoring task during the study phase. The series of digits began $3 \mathrm{sec}$ before the presentation of the first study pair and ended $3 \mathrm{sec}$ after the last presentation. The subjects wrote down the total number of even digits that had been presented as soon as the tape ended.

\section{Results and Discussion}

The mean proportion of "old" responses in the four test conditions and their $95 \%$ within-subjects confidence intervals are shown for the two study conditions in Figure 2. The results are quite different from those obtained in Experiment 1 and are similar, instead, to those that were obtained for faces in previous experiments. There was a significant main effect of test condition $[F(3,138)=36.48$, $\left.M S_{\mathrm{e}}=0.09, p<.01\right]$. There was not a main effect of study condition $[F(1,46)=.17]$, indicating approximately equal overall performance in the simultaneous- and sequentialstudy conditions. We analyzed digit-monitoring performance to ensure that this equality of performance was not the result of a differential tradeoff between word encoding and digit monitoring. There was no statistical evidence for differential digit-monitoring performance across the two study conditions. The correct number of even digits was 24 ; the mean numbers reported were 


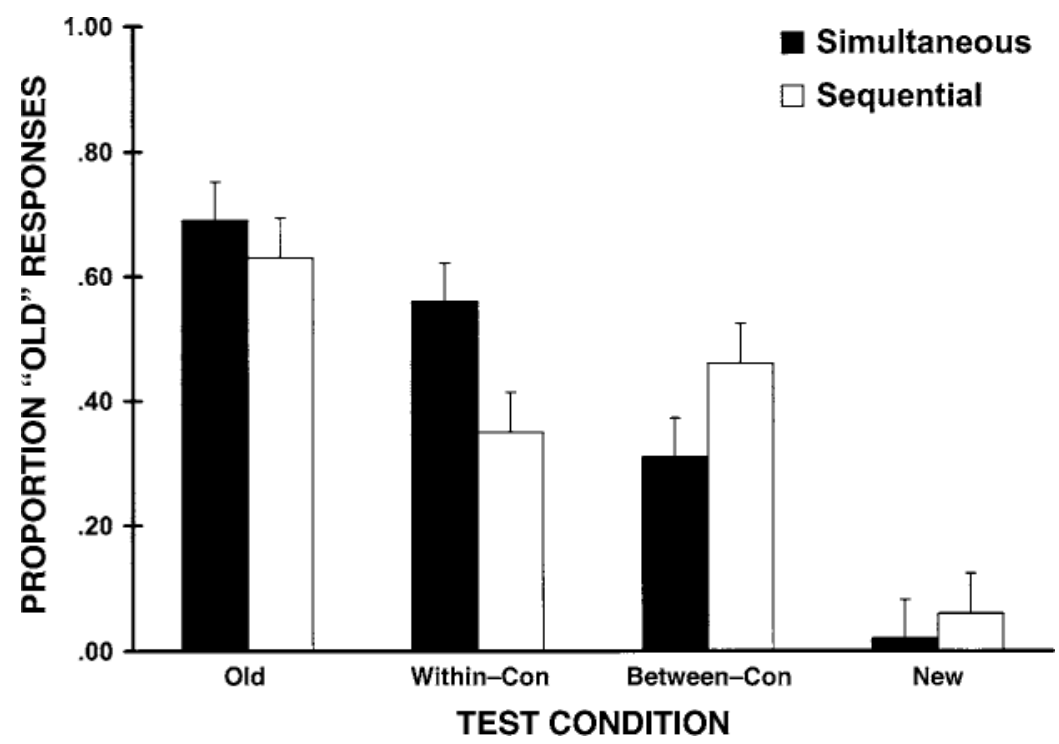

Figure 2. Results of Experiment 2: Proportion of "old" responses in each of the four test conditions for the two study conditions. The error bars show the $95 \%$ withinsubjects confidence intervals (G. R. Loftus \& Masson, 1994). Within-Con and Between-Con refer to the within-pair and between-pair conjunction conditions, respectively.

22.71 and 23.54 in the simultaneous- and sequentialstudy conditions, respectively $[t(46)=1.28]$. Ten subjects in the simultaneous condition correctly reported the number of digits, and 11 subjects in the sequential condition did so.

As in Experiment 1, the subjects in the simultaneousstudy condition made more false alarms to within-pair conjunction stimuli than to between-pair conjunction stimuli $\left[F(1,23)=4.60, M S_{\mathrm{e}}=0.16, p<.05\right]$. However, there clearly were not more within-pair than betweenpair conjunction errors in the sequential-study condition $\left[F(1,23)=1.501, M S_{\mathrm{e}}=0.09, p=.23\right]$; in fact, there were numerically more between-pair conjunction false alarms in this condition. The study condition $\times$ test condition interaction was significant $\left[F(1,46)=5.34, M S_{\mathrm{e}}=\right.$ $0.125, p<.05],{ }^{1}$ supporting the idea that when rehearsal is prevented parts of separate, simultaneously presented words are interassociated in memory, and parts of separate, sequentially presented within-pair words are not interassociated.

It is worth noting that in the present experiment the subjects in the sequential-study condition made numerically more false alarms in the between-pair conjunction condition than in the within-pair conjunction condition. Although the difference did not approach significance, it may imply a tendency for more between-pair than withinpair errors in this condition. However, in the absence of additional evidence for such a trend, we tend to attribute the difference to statistical noise, because there are no reasonable theories that we can think of that would predict more errors in the between-pair condition than in the within-pair condition. We have published detailed inves- tigations of the effects of study-list distance on conjunction errors, and in every case we have found that conjunction errors decrease, rather than increase, as the distance between the parent items on the study list increases (see, e.g., Hannigan \& Reinitz, 2000). Moreover, we have published numerous experiments comparing sequential- and simultaneous-study conditions using face stimuli, and we have repeatedly found more withinpair than between-pair conjunction errors in the latter condition and not in the former (e.g., Reinitz \& Hannigan, 2001). Thus, it seems unlikely that there is a real trend toward more between-pair than within-pair conjunction errors, although additional research is needed to completely rule out this interesting possibility.

The results of Experiment 2 lead to two important conclusions. First, the Experiment 1 finding of more within-pair than between-pair conjunction errors in the sequential-study condition resulted from the simultaneous conscious awareness of the two within-pair words during the study phase. When prolonged working memory activation of sequentially presented words was prevented by the digit-monitoring task, the pattern of results changed markedly. These findings are consistent with our previous assertions regarding the role of working memory in binding. When subjects are concurrently aware of two stimuli, the parts of those stimuli tend to be remembered together, and it is hard for subjects to assign the parts to the stimuli that had originally contained them.

Second, these results support the generality of our previous findings when faces were used as stimuli. This is important because it suggests that working memory has 
a uniform binding function across stimulus types. When items are presented sequentially and rehearsal is prevented (either because of perceptual complexity, in the case of faces, or because of the presence of a secondary task, in the case of words), subjects no longer have greater difficulty rejecting within-pair conjunction stimuli relative to between-pair conjunction stimuli. When subjects are concurrently aware of multiple items (because they are presented simultaneously or because one item is rehearsed whereas the other is presented), there are more within-pair than between-pair conjunction errors.

Finally, regarding attentional effects, we note that overall sensitivity to the studied words was lower in Experiment 2 than in Experiment 1. For instance, the hit rate dropped from about .9 in Experiment 1 to about .65 in Experiment 2. In addition, there were more false alarms overall in Experiment 2 than in Experiment 1. These effects are not surprising, considering that an attentional load was introduced in Experiment 2. In other words, there appear to be two separate effects of dividing attention. One is to prevent rehearsal, so that only one word at a time was resident in working memory in the sequentialstudy condition. The other was to generally reduce encoding efficiency, so that performance on old items was reduced and false alarms to foils increased.

\section{EXPERIMENT 3}

The results of Experiments 1 and 2 show that when subjects are simultaneously aware of two compound words, they are especially likely to endorse conjunction words comprising their parts. In Experiment 3, we tested whether the effects that we have observed to occur in recognition also occur in recall. This is important for several reasons. First, if we are correct in asserting that concurrent inclusion in working memory leads to the formation of associations between parts of items so that they are subsequently difficult to disentangle into individual memories, then this effect should be observed across memory tests.

Second, it is currently controversial whether conjunction errors occur in recall or are instead restricted to recognition tests. To date, the only published demonstration of such errors in recall is from Reinitz et al. (1992), who had subjects study simple sentences such as "The doctor saw the bear" and "The lawyer saw the goat." Conjunction responses (e.g., "The doctor saw the goat") were fairly common on a subsequent recognition test; however, the arbitrary and nonintegral nature of their stimuli raises the possibility that the results might be restricted to the specific stimuli that were used in that study. Errors on the compound word stimuli used here would provide a more general demonstration that conjunction errors occur in recall.

Finally, we have argued that the high rate of withinpair conjunction errors following simultaneous-study presentations reflects patterns of binding in episodic memory, rather than familiarity. Although some models of familiarity provide a mechanism for familiarity-based context effects in both recall and recognition (see, e.g., Gillund \& Shiffrin, 1984), many theorists have argued that familiarity is relatively insensitive to context. To the extent that this is true, all of the parts of previously presented items should give rise to about the same amount of familiarity. If familiarity alone were responsible for false alarms to conjunction items, then one might expect an equal number of false alarms to within-pair and betweenpair conjunction items, since both types of items contain the same number of old parts and, thus, should give rise to equal familiarity. If we were to find that study condition specifically influenced recall responses, this would provide additional evidence that patterns of working memory processing during the study phase specifically influenced subsequent conscious recollection.

The study phase of Experiment 3 was identical to that of Experiment 1; however, instead of receiving a recognition test, subjects were simply asked to write down all of the words that they could remember from the study phase.

\section{Method}

Subjects. Forty-eight Boston University undergraduates participated for credit in their introductory psychology classes. They were run in 16 groups of 3 subjects each. None of them had participated in either of the previous experiments.

Stimuli and Apparatus. The same stimuli and apparatus used in Experiment 1 were again used here.

Design and Procedure. The procedure of the study phase was identical to the Experiment 1 study procedure, and the same study orders were used. Following the 10-min mental rotations test, the subjects were simply asked to write down, in any order, all of the words that they were sure they could remember from the study phase. The subjects were given $5 \mathrm{~min}$ for the recall task.

\section{Results and Discussion}

Table 2 shows the mean number of correct responses, within-pair conjunction errors, and between-pair conjunction errors in the two study conditions. In keeping with previous experiments, only responses relevant to the middle five study positions were analyzed. The maximum number of correct responses was therefore 10, and the maximum number of conjunction errors was 2 in each conjunction condition. The subjects never made betweenpair conjunction errors in recall; in fact, none of the subjects in the sequential-study condition made any conjunction errors. However, the mean frequency of within-pair conjunction errors was .38 in the simultaneous-study

Table 2

Mean Correct Responses, Within-Pair Conjunction Errors, and Between-Pair Conjunction Errors in the Two Study Conditions

\begin{tabular}{cccc}
\hline & & \multicolumn{2}{c}{ Errors } \\
\cline { 3 - 4 } Study Condition & Correct & W-Con & B-Con \\
\hline Simultaneous & 3.75 & 0.38 & 0 \\
Sequential & 5.29 & 0 & 0 \\
\hline
\end{tabular}

$\overline{\text { Note-W-Con, within-pair conjunction condition; B-Con, between- }}$ pair conjunction condition. 
condition. Since each subject had only two opportunities to make these errors, this indicates that simultaneousstudy subjects made within-pair errors about $20 \%$ of the time when such errors were possible. Analyses using $t$ tests showed that the subjects made significantly more within-pair conjunction errors than between-pair conjunction errors in the simultaneous-study condition $[t(23)=$ $3.19, p<.01]$ and made significantly more within-pair conjunction errors in the simultaneous-study condition than in the sequential-study condition $[t(46)=3.19, p<$ $.01]$. Given that there were no conjunction errors in some of the conditions, we also used nonparametric analyses to test for these effects. A sign test confirmed that the subjects made more within-pair conjunction than betweenpair conjunction errors in the simultaneous-study condition $(p<.01)$, and Fisher's exact test showed that they made more within-pair errors in the simultaneous- than in the sequential-study condition $(p<.01)$. The results demonstrate that the effects of simultaneous stimulus presentation that were observed in the previous experiments generalize to recall, and support the notion that those effects take place specifically on conscious remembering.

The subjects in both study conditions sometimes made another type of error, in which part of a previously studied word was combined with a new (unstudied) part. The average frequencies of such intrusion errors across subjects were 0.42 in the simultaneous-study condition and 0.38 in the sequential-study condition; this difference did not approach significance. It is important to note that these frequencies are not comparable to the mean frequencies of the conjunction errors. The reason is that there were only two opportunities for each subject to make each type of conjunction error. However, any previously studied word part could conceivably be recombined with a new word part. Since 10 words were presented across the relevant study positions, there were 20 opportunities for intrusion errors to occur. Nonetheless, the occurrence of intrusion errors indicates that parts of some previously studied words seem to have been "free floating" in memory for both study conditions; however, those parts tended to be recollected with parts of paired items in the simultaneous-study condition only.

Our experiments are consistent in indicating that when words are presented simultaneously, subjects have a tendency to falsely recognize (Experiments 1 and 2) and falsely recall (Experiment 3 ) new words comprised of their parts. However, it is interesting to note that the recall results diverge from the recognition results in an important way. Specifically, the subjects in the sequential-study condition made more within-pair than between-pair conjunction errors on the Experiment 1 recognition test, but not on the Experiment 3 recall test, despite the fact that the study procedure was identical in the two cases. It therefore appears that the deliberative recollective processes involved in recall can serve to insulate people from making within-pair conjunction errors for sequentially presented (but not for simultaneously presented) words. This may indicate that parts of sequentially presented within-pair words are not as strongly associated as are parts of simultaneously presented within-pair words, perhaps because perceptual representations tend to have greater strength than memorial representations. As a result, simultaneously perceiving two words would produce stronger between-part associations than would be observed when one word was perceived and another was remembered. Relatively small differences in associative strength would not substantially impact responding in a recognition context, because two associated items are presented together in conjunction lures, leading to substantial summed associative strength. However, if the subject recalls part of a previously presented word, substantial associative strength would be needed to activate the other part. In this case, subjects may activate the other part of the original compound, leading to a correct response, or they may fail to activate any previously presented component, leading to an omission or an intrusion error. High associative strength between parts of separately studied words would be necessary in order to activate the other component of a conjunction item. To the extent that between-item associations are somewhat stronger for simultaneously presented items than for sequentially presented paired items, one would therefore expect more conjunction errors for simultaneously presented words than for sequentially presented paired words in recall, but approximately equal conjunction errors for those conditions in recognition.

Other factors undoubtedly contribute to the differences we observed between recall and recognition. For instance, familiarity plays a much larger role in recognition than in recall. Familiarity engendered by old word components presented during the recognition test may reduce subjects' tendencies carefully to think back on events that occurred during the study phase relative to recall subjects, who are not presented with old familiar components and, so, must think back more deliberatively. This may lead to increased rejections of conjunctions that come to mind, particularly when those conjunctions are from sequentially presented words whose parts are not bound together in memory. Finally, the difference may be partly attributable to different overall performance levels in the two study conditions of Experiment 3: The subjects recalled significantly more words correctly in the sequential condition than in the simultaneous-study condition $[t(46)=-2.43, p<.05]$, and it is possible that this increase in recall helped them to reject conjunction items that came to mind during the test.

In any case, the results of Experiment 3 clearly demonstrate that conjunction errors occur in recall, and are generally consistent with our arguments concerning the role of working memory processing in binding. Most importantly, they demonstrate that when words are studied simultaneously, subjects tend to mix up their parts during recall. 


\section{GENERAL DISCUSSION}

\section{Summary}

In previous experiments, we presented faces either simultaneously or sequentially and found that subjects made more false alarms to within-pair conjunction faces than to between-pair conjunction faces in the simultaneousstudy condition and not in the sequential-study condition. In Experiment 1, using words as stimuli, there were more within-pair than between-pair conjunction errors in both study conditions. However, when the subjects performed a digit-monitoring task during the study phase (Experiment 2), the pattern of results was essentially identical to the pattern that we obtained for faces. The implication is that face-word conjunction-error differences simply reflect the fact that words are much easier to rehearse than faces. As a result, sequentially presented words may be simultaneously present in working memory. When rehearsal is prevented, the same binding mechanisms seem to determine the pattern of recognition errors for both stimulus types.

Experiment 3 showed that the subjects recalled conjunctions of simultaneously presented words about $20 \%$ of the time when such conjunction errors were possible. This demonstrates that a recall-based mechanism can produce conjunction errors, and is consistent with the proposal that patterns of working memory processing during the study phase specifically influence conscious remembering later on.

\section{Role of Working Memory in Binding}

The effects of simultaneous- versus sequential-study presentations that we have reported support the proposal that working memory processes mediated by prefrontal cortex determine how parts of an experience are bound together into a unified memory representation. This contribution to the binding process is consistent with, and probably related to, the more general integrative functions that are widely attributed to frontal lobe processing.

Binding is the result of an interaction between the hippocampus and the prefrontal cortex. In consistency with previous proposals by Köhler and Moscovitch (1997) and Moscovitch (2000), we believe that this interaction is best characterized as a compose-and-bind mechanism. The specific role of prefrontal (working memory) processing is probably to compose a potential long-termmemory representation by integrating various aspects of an experience into a transient, conscious memory structure. These composed memories serve as input to the hippocampus, which may bind them into durable representations in episodic memory by forming associations between the various integrated components. According to this proposal, binding failures may occur when there is impoverished prefrontal processing (as in the cases of aging and divided attention). Binding failures may also occur despite robust prefrontal processing when there is impoverished mediotemporal processing (as in the case of anterograde amnesia resulting from mediotemporal damage).

Our results clearly demonstrate that the strength of associations formed during the binding process vary on the basis of several factors. The recall data suggest that the associations formed between parts of separate stimuli are less strong in a sequential-study condition than in a simultaneous-study condition even when subjects intentionally rehearse the first item while viewing the second one. Additionally, all of the experiments demonstrate that associations between parts of single study items are stronger than associations between parts of separate study items. In all cases, the subjects recognized or recalled more old stimuli than conjunction stimuli. This may be because the subjects were aware of both the paired items together and the individual stimuli. In some extreme cases, it appears that there is a binding failure such that parts are encoded but interpart associations are not. This occurs in the case of severe hippocampal damage (see, e.g., Kroll et al., 1996; Reinitz et al., 1996) and when attention is focused on a very demanding secondary task (Reinitz et al., 1994).

By our explanation, subjects may have difficulty discriminating old items from conjunction items for two general reasons. First, binding may be impaired, as is the case in hippocampal damage or divided attention. In these cases, we propose that "old" responses to both old and conjunction items are driven by recognition of individual stimulus components. Both types of stimuli have the same number of old components, so the two stimuli receive approximately equal "old" responses. Second, all of the parts of similar items that are concurrently present in working memory may become bound together in episodic memory. In this case, all of those parts have a tendency to be remembered together. As a result, it is difficult to discriminate between an old item and a withinpair conjunction item, but it is relatively easy to reject a between-pair conjunction item. Thus, discrimination problems occur when binding is impaired or when binding is indiscriminate.

\section{Alternate Explanations}

As is almost always the case in memory research, it is possible to devise alternate explanations for our results. For instance, numerous authors have proposed that memory conjunction errors often result from familiaritybased processes rather than from binding. The idea is that parts of old items will give rise to familiarity. As the number of old parts that are present in a stimulus increases, so will the familiarity that is engendered by the stimulus. If subjects failed to remember the original stimuli that contained those parts, then this familiarity would produce a tendency to respond that conjunction stimuli are old. In accordance with this explanation, Jones and Jacoby (2001) showed that manipulations known to influence recollection and not familiarity tend to decrease hits to old items, but not false alarms to conjunc- 
tion items, in recognition tests. Similarly, Rubin, Van Petten, Glisky, and Newberg (1999) showed that eventrelated potentials (ERPs) accompanying false alarms to conjunction stimuli were more similar to ERPs accompanying false alarms to new stimuli than to those accompanying hits to previously presented stimuli (which would likely be based on recollection). However, in neither of those studies were stimuli presented in pairs, and our results imply that simultaneous study is the factor that results in increased within-pair conjunction errors. More generally, it should be noted that experiments cited in support of familiarity-based explanations have tended to employ manipulations that reduced "old" responses to old items but not to conjunction items. This decrease in responding to old items is consistent with the idea that those manipulations reduced recollection but not familiarity. In the present experiments, manipulations that we expected to increase between-feature binding led to an increase in within-pair conjunction errors, leaving responses to old items unaffected. This pattern is predicted by binding-based explanations and is inconsistent with many familiarity-based explanations.

One version of a familiarity-based explanation can account for our patterns of recognition results. There is evidence that familiarity is mediated in part by priming and that simultaneously studied words prime one another much more than do sequentially presented words (GoshenGottstein \& Moscovitch, 1995). Thus, parts of simultaneously presented words might prime one another more than parts of separately presented words, leading to greater summed familiarity for within-pair conjunction words than for between-pair conjunction words. Although this interesting explanation cannot be ruled out, there are problems with it. First, Reinitz and Demb (1994) and Reinitz et al. (1996) showed that although there is strong priming for previously studied compound words, there is no priming for conjunction words made by recombining parts of previously studied words. Thus, there is no reason to expect any priming at all for conjunction words, regardless of whether they are made from parent items that had been studied together or separately. We agree with other authors that memory conjunction errors sometimes result from familiarity-based processes; however, it seems likely that the results of the present experiments reflect binding processes. As is the case for most complex mental phenomena, memory conjunction errors may be produced by multiple underlying mechanisms. The familiaritybased and binding-based explanations are not mutually exclusive, and both mechanisms probably sometimes produce memory conjunction errors.

A contributing factor that cannot be ruled out in the present study is that the subjects may have generated conjunction items during the study phase and then remembered generated items as items that had previously been presented. We think that this explanation is unlikely for a number of reasons. First, most of the subjects expressed surprise during the debriefing, when we explained the nature of the memory errors for which we were testing, and few subjects reported noticing that parts of separate stimuli could be recombined (note that within-pair conjunctions were possible on only two study trials). Second, the pattern of results for words was identical to the pattern observed for faces when rehearsal was prevented. It seems highly unlikely that the subjects generated mental examples of recombined items as they studied the faces. Given the similarity of the results for the two stimulus types, it is reasonable to assume that similar processes gave rise to them. Finally, this explanation predicts that the subjects should have recalled conjunctions equally often in the two study conditions of Experiment 3; however, the subjects recalled conjunction items only when words were studied simultaneously. Nonetheless, an investigation of the specific thoughts that go on while subjects study the stimuli would be both interesting and useful.

\section{Final Comments}

We close by pointing out three general implications of our results. First, a salient difference between unfamiliar faces and words is that words have unitary semantic representations, whereas unfamiliar faces must be remembered as visual information. Nonetheless, when rehearsal was prevented, we obtained a pattern of errors for words identical to that which we have repeatedly obtained for faces. This implies that the patterns of results that we have obtained are very general. Additionally, the high false alarm rates that we obtained for words in our experiments indicate that the existence of an underlying semantic representation does little to help prevent conjunction errors in recognition, even when subjects study relatively few words and are tested after only a few minutes. Indeed, Hannigan and Reinitz (2003) provided evidence that semantic information acquired in one episode can migrate in memory in such a way that subjects later remember that information as applying to a different episode. Together, these findings indicate the very broad generality of these errors and suggest that they will occur whenever memories are comprised of discrete units, regardless of the code of those units.

Second, our results imply that whenever two items are concurrently active in working memory, their parts may be bound together. Experiment 2 showed that this is true even when a previously experienced item is active while a new item is being studied. This finding may have general consequences for understanding memory illusions. For instance, in the standard misinformation effect paradigm (see, e.g., E. F. Loftus et al., 1978) a question serves to reactivate the original event while providing misleading information. As a result, both the original event and the misinformation are active in working memory, and therefore may be bound together.

Finally, we point out that these errors have real-world relevance. Parts of items that occur together are highly confusable, whether those items are faces or words. This result implies that parts of items that are simultaneously present at a crime scene (e.g., faces, license plates) may 
be especially prone to be miscombined in a witness's memory. Other studies may have underestimated the frequency of conjunction errors for words, because they lacked a within-pair condition. Moreover, we have shown that there may also be a high conjunction error rate for words presented in rapid sequence rather than simultaneously, in contrast to faces presented in the same way. Previous research has demonstrated that subjects tend to be highly confident of their "old" responses to conjunction stimuli (Reinitz et al., 1992). Furthermore, we recently demonstrated that when faces are studied in a simultaneous-study condition, it becomes increasingly difficult for subjects to discriminate within-pair conjunction items from truly old items as the retention interval increases (Hannigan \& Reinitz, 2000). Criminal cases often go to trial many months after a crime has been committed. These factors may therefore give rise to confident eyewitness reports that are actually conjunctions of previously experienced stimuli.

\section{REFERENCES}

Albert, W., Reinitz, M. T., Beusmans, J., \& Gopal, S. (1999). Role of attention in spatial learning during simulated route navigation. Environment \& Planning A, 31, 1459-1472.

Bower, G. H., Black, J. B., \& Turner, T. J. (1979). Scripts in memory for texts. Cognitive Psychology, 11, 177-220.

Braver, T. S., Barch, D. M., Keys, B. A., Carter, C. S., Cohen, J., Kaye, J. A., Janowsky, J. S., Taylor, S. F., Yesavage, J. A., MuMenthaler, M. S., Jagust, W. J., \& Reed, B. R. (2001). Context processing in older adults: Evidence for a theory relating cognitive control to neurobiology in healthy aging. Journal of Experimental Psychology: General, 130, 746-763.

BROWN, R., \& MCNEILL, D. (1966). The "tip of the tongue" phenomenon. Journal of Verbal Learning \& Verbal Behavior, 5, 325-337.

Chalfonte, B. L., \& Johnson, M. K. (1996). Feature memory and binding in young and older adults. Memory \& Cognition, 24, 403-416.

Chandler, C. C., \& Gargano, G. J. (1998). Retrieval processes that produce interference in modified forced-choice recognition tests. Memory \& Cognition, 26, 220-231.

CoHEN, N. J., \& EICHENBAUM, H. (1993). Memory, amnesia, and the hippocampal system. Cambridge, MA: MIT Press.

Gillund, G., \& Shiffrin, R. M. (1984). A retrieval model for both recognition and recall. Psychological Review, 91, 1-67.

Goshen-GotTstein, Y., \& Moscovitch, M. (1995). Repetition priming effects for newly formed associations are perceptually based: Evidence from shallow encoding and format specificity. Journal of Experimental Psychology: Learning, Memory, \& Cognition, 21, 1249-1262.

HANNIGAN, S. L., \& REINITZ, M. T. (2000). Practical factors that influence the likelihood of false recognition: Influences of temporal factors on memory conjunction errors. Applied Cognitive Psychology, 14, 309-321.

HANNIGAN, S. L., \& ReINITZ, M. T. (2001). A demonstration and comparison of two types of inference-based memory errors. Journal of Experimental Psychology: Learning, Memory, \& Cognition, 27, 931940.

Hannigan, S. L., \& Reinitz, M. T. (2003). Migration of objects and inferences across episodes. Memory \& Cognition, 31, 434-444.

Hintzman, D. L. (1988). Judgments of frequency and recognition memory in a multiple-trace memory model. Psychological Review, 95, 528-551.

IidAKa, T., Anderson, N. D., Kapur, S., CAbeza, R., \& Craik, F. I. M. (2000). The effect of divided attention on encoding and retrieval in episodic memory revealed by positron emission tomography. Journal of Cognitive Neuroscience, 12, 267-280.
Jones, T. C., \& JACOBY, L. L. (2001). Feature and conjunction errors in recognition memory: Evidence for dual-process theory. Journal of Memory \& Language, 44, 82-102.

KöHLER, S., \& Moscovitch, M. (1997). Unconscious visual processing in neuropsychological syndromes: A survey of the literature and an evaluation of models of consciousness. In M. D. Rugg (Ed.), Cognitive neuroscience: Studies in cognition (pp. 305-373). Cambridge, MA: MIT Press.

Kroll, N. E. A., Knight, R. T., Metcalfe, J., Wolf, E. S., \& TulVING, E. (1996). Cohesion failure as a source of memory illusions. Journal of Memory \& Language, 35, 176-196.

Loftus, E. F., Miller, D. G., \& BURNS, H. J. (1978). Semantic integration of verbal information into a visual memory. Journal of Experimental Psychology: Human Learning \& Memory, 4, 19-31.

LoFTUS, G. R., \& MAsson, M. E. J. (1994). Using confidence intervals in within-subject designs. Psychonomic Bulletin \& Review, 1, 476-490.

McClelland, J. L., \& Rumelhart, D. E. (1985). Distributed memory and the representation of general and specific information. Journal of Experimental Psychology: General, 114, 159-188.

METCALFe, J. (1990). Composite holographic associative recall model (CHARM) and blended memories in eyewitness testimony. Journal of Experimental Psychology: General, 119, 145-160.

Moscovitch, M. (2000). Theories of memory and consciousness. In E. Tulving and F. I. M. Craik (Eds.), The Oxford handbook of memory (pp. 609-625). New York: Oxford University Press.

MuRdock, B. B. (1982). A theory of the storage and retrieval of item and associative information. Psychological Review, 88, 93-134.

ReINITZ, M. T. (2002). Illusions of memory. Comments on Theoretical Biology, 6, 411-430.

Reinitz, M. T., \& Demb, J. B. (1994). Implicit and explicit memory for compound words. Memory \& Cognition, 22, 687-694.

Reinitz, M. T., \& Hannigan, S. L. (2001). Effects of simultaneous stimulus presentation and attention switching on memory conjunction errors. Journal of Memory \& Language, 44, 206-219.

Reinitz, M. T., Lammers, W. J., \& Cochran, B. P. (1992). Memoryconjunction errors: Miscombination of stored stimulus features can produce illusions of memory. Memory \& Cognition, 20, 1-11.

ReINITZ, M. T., Morrissey, J., \& DEMB, J. B. (1994). The role of attention in face encoding. Journal of Experimental Psychology: Learning, Memory, \& Cognition, 20, 161-168.

ReINITZ, M. T., Verfaellie, M., \& Milberg, W. (1996). Memory conjunction errors in normal and amnesic subjects. Journal of Memory \& Language, 35, 286-299.

Rubin, S. R., VAn Petten, C., Glisky, E. L., \& Newberg, W. M. (1999). Memory conjunction errors in younger and older adults: Event-related potential and neuropsychological data. Cognitive Neuropsychology, 16, 459-488.

Schacter, D. L., KASZniaK, A. W., Kinlstrom, J. F., \& Valdiserri, M. (1991). The relation between source memory and aging. Psychology \& Aging, 6, 559-568.

STRONG, E. K. (1912). The effect of length of series on recognition memory. Psychological Review, 19, 447-462.

Underwood, B. J., KaPelaK, S. M., \& MALMI, R. A. (1976). Integration of discrete verbal units in recognition memory. Journal of Experimental Psychology: Human Learning \& Memory, 2, 293-300.

Zechmeister, E. B., \& McKillip, J. (1972). Recall of place on the page. Journal of Educational Psychology, 63, 446-453.

\section{NOTE}

1. This $F$ value is from a 2 (study condition) $\times 2$ (test condition) ANOVA that excluded test conditions that were irrelevant to the hypothesis. The study condition $\times$ test condition interaction was also significant in the $2 \times 4$ ANOVA, which included all of the test conditions.

(Manuscript received June 10, 2003; revision accepted for publication November 5, 2003.) 